\title{
DEUX NOUVELLES ONCHOCERQUES NODULAIRES CHEZ DES BOVIDÉS SAUVAGES, EN HAUTE-VOLTA
}

\author{
par A. M. DENKÉ* et O. BAIN**.
}

\begin{abstract}
RÉSUMÉ. Découverte, dans la région d'onchocercose humaine du Bassin de la Volta, de deux nouvelles onchocerques chez des Bovidés sauvages : $O$. schulzkeyi n. sp, parasite d'Hippotagrus equinus, $O$. hamoni n. sp, parasite de Kobus (A.) kob, toutes deux situées dans des nodules, respectivement dermiques et sous-cutanés ; chez ces deux espèces, la cuticule de la $q$ est caractérisée par l'absence de côtes saillantes. Ces 2 nouvelles espèces appartiennent à la lignée d'O. volvulus et des onchocerques nodulaires des Bovinés. Il est donc possible que, comme dans le cas d'O. ochen$g i$, ces espèces puissent fausser l'évaluation du Potentiel Annuel de Transmission utilisé dans l'étude épidémiologique de l'onchocercose humaine.
\end{abstract}

\section{Two new nodular Onchocerca spp. in wild Bovidae, in Upper Volta.}

SUMMARY. Description of two new Onchocerca from wild Bovidae, O. schulzkeyi n. sp. and O. hamoni n. sp., respectively parasite of Hippotragus equinus and Kobus (A.) kob; both species have been recorded in the Volta region, where human onchocerciasis is prevalent. O. schulzkeyi and $O$. hamoni are located in respectively, dermic and subcutaneous nodules, the females of both species are devoid of cuticular rings. Those two new species belong to the line of $O$. volvulus and nodular Onchocerca of Bovinae. This finding suggests that, as for $O$. ochengi, they can disturb the evaluation of the annual potential of transmission, necessary in the épidemiological studies concerning the human onchocerciasis.

Deux nouvelles onchocerques nodulaires ont été trouvées chez des Bovidae sauvages, en mars 1980, dans la réserve de Comoe, à Folonzo, en Haute-Volta.

\section{1 - Onchocerca schulzkeyi n. sp.}

Hôte : Hippotragus equimus (Desmarest), Hippotraginae.

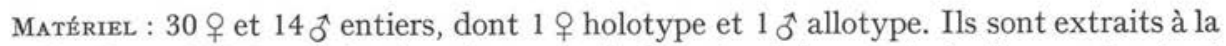
collagénase de 30 nodules dermiques situés dans la région ventrale d'un seul animal (sternum, xyphoïde, ombilic). Les nodules pèsent de 0,4 à $1,5 \mathrm{~g}$ et mesurent 1 à $1,5 \mathrm{~cm}$

* Dr Vétérinaire, B.P. 3374, Lomé, Togo; Consultant OMS, Programme de Lutte contre l'Onchocercose.

** Laboratoire Zoologique des Vers, 43 rue Cuvier, F 75231 Paris Cedex 05

Accepté le 18 novembre 1980. 


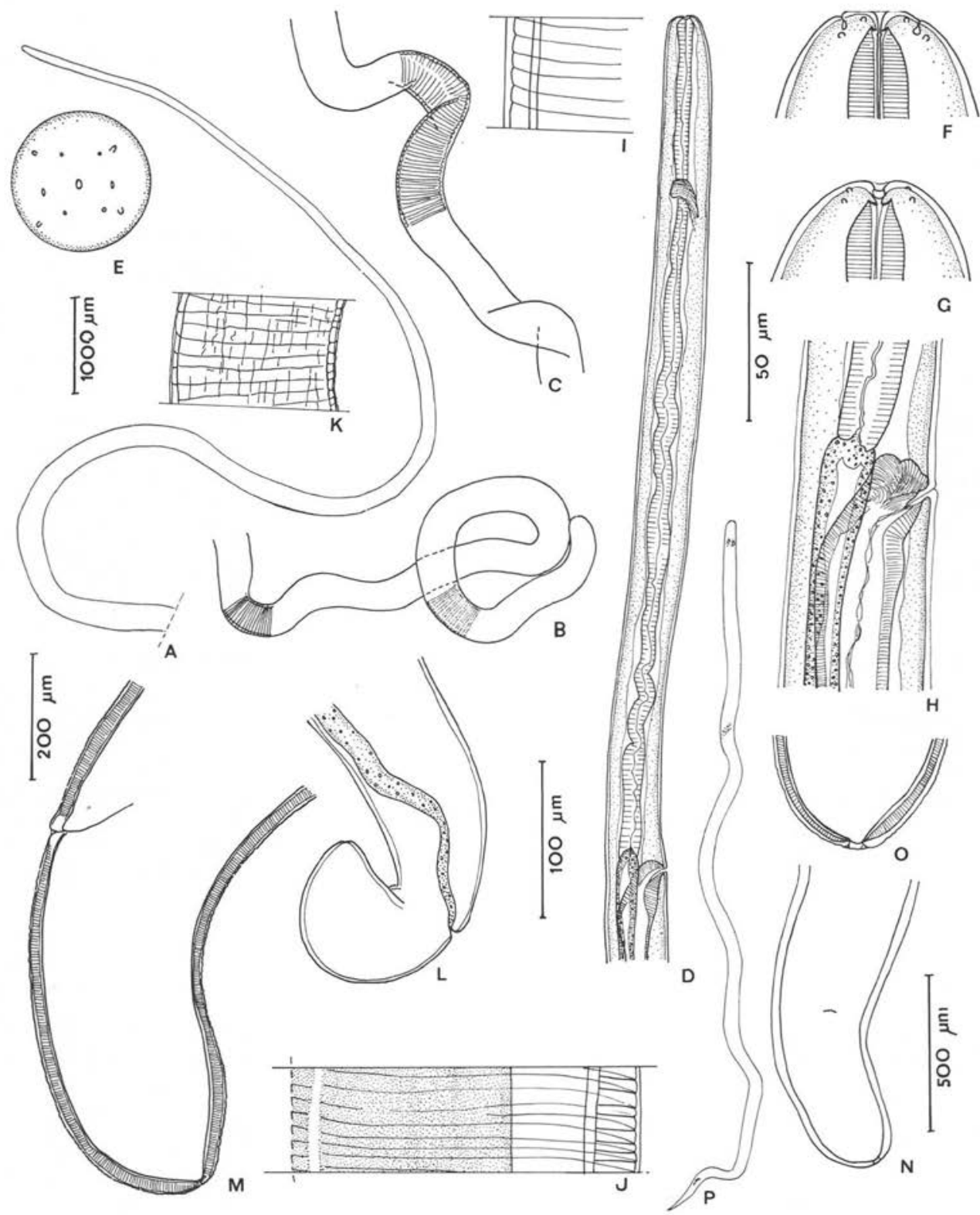

FIG. I. - Onchocerca schulzkeyi n. sp., femelle; A, B, C, : silhouette des régions antérieures, postérieure et moyenne du corps; D : région antérieure, vue latérale, E, F, G : tête, vues apicale, médiane et latérale ; $\mathrm{H}$ : région vulvaire, vue latérale; $\mathrm{I}, \mathrm{J}$, : ornementation cuticulaire du corps à $8 \mathrm{~mm}$ et $24 \mathrm{~mm}$ de l'extrémité antérieure (coupe optique longitudinale) ; $\mathrm{K}$ : cuticule de la région moyenne du corps avec sillons longitudinaux et stries transversales; $\mathrm{L}:$ queue d'une + vue latérale; $\mathrm{M}, \mathrm{N}$ : queue d'une autre $\not$ en vue latérale (stries cuticulaires représentées en coupe optique seulement) et ventrale; $\mathrm{O}$ : extrémité caudale de cette $O$, vue ventrale; $\mathrm{P}$ : microfilaire extraite de l'ovéjecteur $(\mathrm{A}, \mathrm{B}, \mathrm{C}$, ech. rooo $\mu \mathrm{m} ; \mathrm{D}, \mathrm{M}, \mathrm{O}$, éch. $200 \mu \mathrm{m} ; \mathrm{H}$, éch. Ioo $\mu \mathrm{m}$; L. K. N éch. $500 \mu \mathrm{m}, \mathrm{E}, \mathrm{F}, \mathrm{G}, \mathrm{I}, \mathrm{J}, \mathrm{P}$ éch. $50 \mu \mathrm{m})$. 
de diamètre ; ils renferment 1 , parfois 2 (6 cas); un nodule sur deux en moyenne renferme aussi 10 ; $50 \%$ des + ont des microfilaires $(6$ des 16 q à microfilaires ne sont

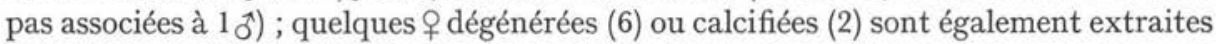
de l'ensemble des nodules.

\section{Description.}

FEMelle : ( fig. I et 2) : ver long d'un mètre et atteignant un demi-millimètre de large ; région antérieure très effilée et à courbures peu accentuées ; ondulations plus serrées dans le reste du corps. Cuticule au milieu du corps formée par 3 couches : couche externe, sans côtes cuticulaires transversales mais creusée irrégulièrement de rides longitudinales discontinues ; couche moyenne plus épaisse, découpée transversalement par des stries (au faible grossissement les rides de la couche externe et les stries de la couche moyenne donnent un aspect quadrillé à la cuticule du corps) ; couche interne mince. Cordes latérales hypertrophiées, à 4 files longitudinales de noyaux ; musculature atrophiée, sauf dans la région antérieure du ver, et montrant une légère asymétrie bilatérale.

Esophage mince sur toute sa longueur et non divisé. Vulve près de la jonction œsophage-intestin, un peu en avant ou un peu en arrière ; portion impaire de l'ovéjecteur longue (4850 $\mu \mathrm{m}$ chez une +). Grande queue, droite ou courbée sur la face dorsale, à phasmides terminales très rapprochées de l'axe médian.

Dimensions : ㅇ holotype : corps longs de $100 \mathrm{~cm}$ environ, large de $100 \mu \mathrm{m}$ à la jonction œsophage-intestin, de $550 \mu \mathrm{m}$ au maximum au milieu du corps et de $250 \mu \mathrm{m}$ au niveau de l'anus ; épaisseur de la cuticule de 18-20 $\mu \mathrm{m}$ à $2 \mathrm{~cm}$ de la tête, de 25-30 $\mu \mathrm{m}$ à mi-corps ; stries cuticulaires espacées de $35-45 \mu \mathrm{m}$; anneau nerveux à $250 \mu \mathrm{m}$ de l'apex; œsophage long de $1180 \mu \mathrm{m}$; vulve à $1020 \mu \mathrm{m}$ de l'apex; queue longue de $600 \mu \mathrm{m}$.

3 ㅇ paratypes : corps long de $100 \mathrm{~cm}$ environ, large de 100,150 , et $90 \mu \mathrm{m}$ à la fin de l'œsophage et de $500,550,550 \mu \mathrm{m}$ à mi-corps; cuticule épaisse de 20 à $55 \mu \mathrm{m}$ à mi-corps et stries espacées de 38 à $68 \mu \mathrm{m}$ pour l'ensemble des 3 ; ; anneau nerveux à 250,250 , et $215 \mu \mathrm{m}$ de l'apex; œsophage long de 1340,1250 et $1200 \mu \mathrm{m}$; vulve à 1370, 1500 et $1100 \mu \mathrm{m}$ de l'apex ; queue longue de $515 \mu \mathrm{m}$, (cassée) et $610 \mu \mathrm{m}$.

MãLE (fig. 3) : cuticule striée transversalement; œsophage non divisé ou divisé ; 11 à 14 papilles caudales disposées sur 2 lignes latéro-ventrales de façon assez variable ; papille impaire précloacale non observée ; phasmides à la base de la $9^{\mathrm{e}}$ paire de papilles transformée en pointes cuticularisées. Spicule droit au talon saillant et à spatule courte. Spicule gauche à pointe simple ou marquée d'une encoche située à mi-longueur de la pointe.

Dimensions : $\widehat{\jmath}$ allotype : corps long de $45 \mathrm{~mm}$, large de $95 \mu \mathrm{m}$, anneau nerveux à $210 \mu \mathrm{m}$ de l'apex ; œsophage long de $1380 \mu \mathrm{m}$, à portion glandulaire de $1060 \mu \mathrm{m}$; spicule gauche et droit longs de 200 et $80 \mu \mathrm{m}$; queue longue de $85 \mu \mathrm{m}$. 

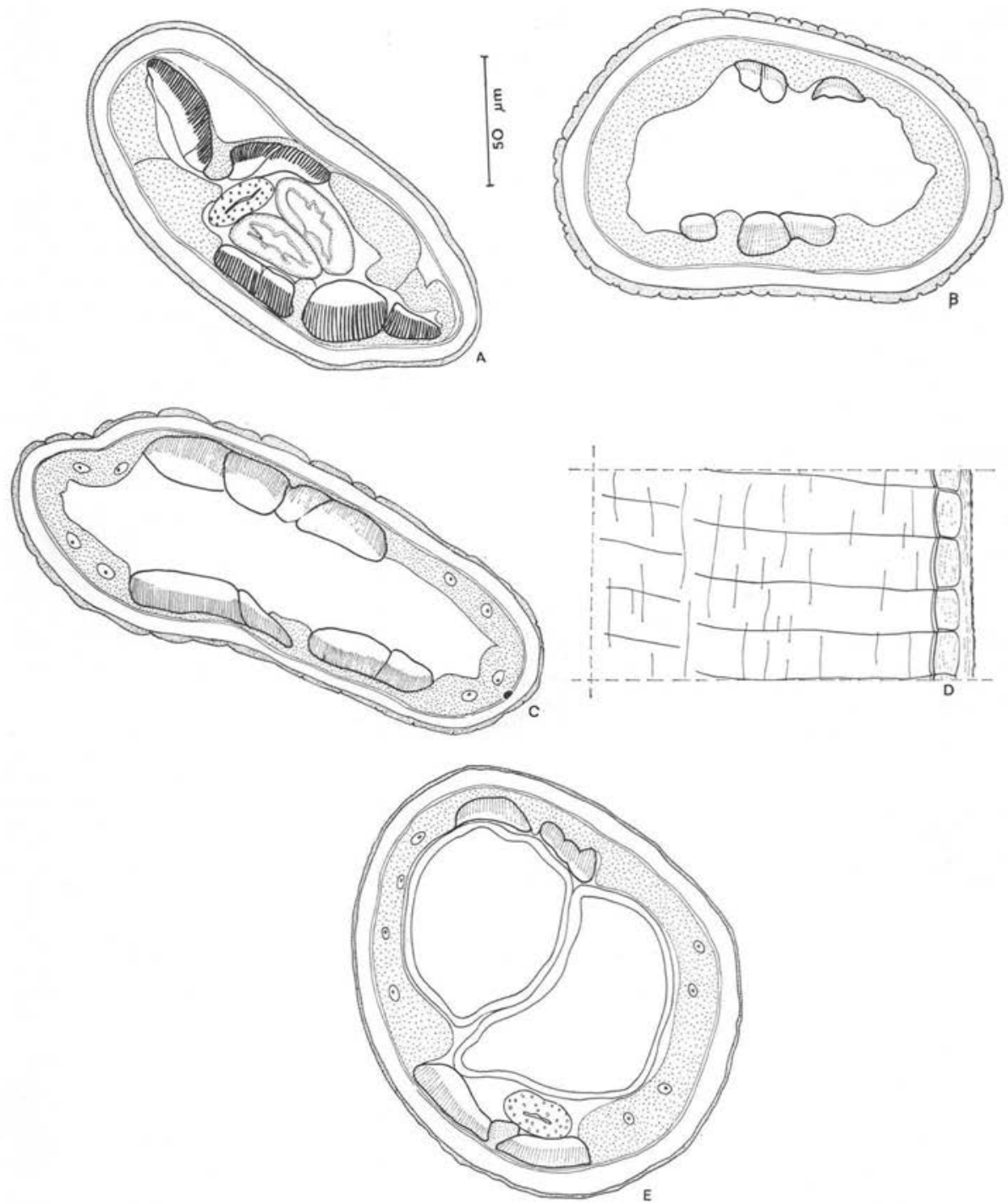

FIG. 2. - A à D, O. schulzkeyi n. sp., 우; A, B, C, coupes transversales d'une $ᄋ$ à $2 \mathrm{~cm}$ de l'apex, dans la région moyenne du corps, et à $\mathrm{I} \mathrm{cm}$ de l'extrémité caudale (en $\mathrm{A}$, les fibrilles musculaires sont plus développées qu'en $\mathrm{B}$ et C) ; D : structure de la cuticule dans la région moyenne du corps, coupe optique longitudinale; Onchocerca hamoni n. sp., 우; E : coupe transversale dans la région moyenne du corps. (A, B, C, D, E, éch. $50 \mu \mathrm{m})$. 


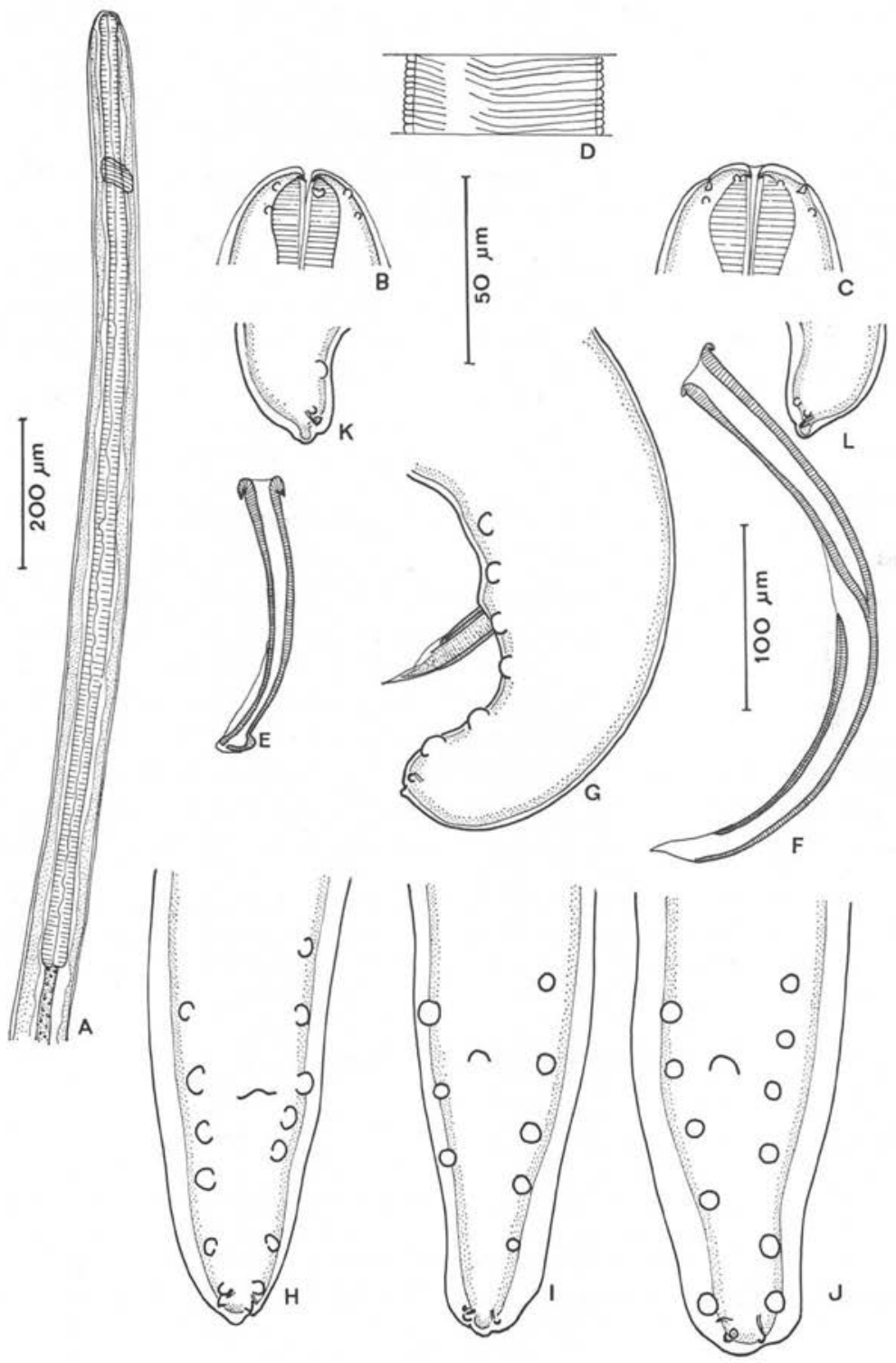

Fig. 3. - Onchocerca schulzkeyi $\mathrm{n}$. sp. $\sigma^{\Uparrow}$; A : région antérieure, vue latérale; B, C : tête, vues latérale et médiane; $\mathrm{D}$ : cuticule, à mi-corps, vue semi-latérale ; $\mathrm{E}$ : spicule droit, vue latérale ; $\mathrm{F}$ : spicule gauche, vue latérale ; $\mathrm{G}$ : queue vue latérale; $\mathrm{H}, \mathrm{I}, \mathrm{J}$, : queues de $3 \mathrm{o}^{\hat{}}$, vue ventrale ; $\mathrm{K}$ et L : extrémité caudale droite et gauche du ${ }_{0}{ }_{\mathrm{H}}$; (A, éch. $200 \mu \mathrm{m} ; \mathrm{D}$ : éch. $100 \mu \mathrm{m} ; \mathrm{E}$ à L éch. 50 rim). 
2 ๙ิ paratypes : corps longs de 49 et $44 \mathrm{~mm}$, larges de 80 et $95 \mu \mathrm{m}$; stries espacées de 4 et $4 \mu \mathrm{m}$; anneau nerveux à 220 et $200 \mu \mathrm{m}$ de l'apex; œsophage long de 1310 et $1180 \mu \mathrm{m}$, non divisé ; spicule long de 185 et $180 \mu \mathrm{m}$ (manche long de 80 et $70 \mu \mathrm{m}$ ); spicule droit long de 78 et $76 \mu \mathrm{m}$; queue longue de 70 et $70 \mu \mathrm{m}$.

Microfilaires ( $f i g . I P$ ). Dans l'ovéjecteur des 우, microfilaires au corps mince, un peu effilé dans la région antérieure ; tête arrondie, à crochet très petit.

Chez la 우 holotype, corps long de 225 à $265 \mu \mathrm{m}(225,232,235,228,229,233,228$, $235,227,237,265 \mu \mathrm{m}$ ) et large de 3,5 à $4 \mu \mathrm{m}$. Sur la microfilaire de $265 \mu \mathrm{m}$, espace céphalique long de $6 \mu \mathrm{m}$, anneau nerveux à $65 \mu \mathrm{m}$ de l'apex, dernier noyau à $15 \mu \mathrm{m}$ de l'extrémité caudale.

\section{Discussion}

Par la morphologie du fourreau épithélio-musculaire (hypertrophie de l'hypoderme et principalement des cordes latérales, atrophie de la musculature) et l'œsophage peu allongé (inférieur à $1500 \mu \mathrm{m}$ ), à portion glandulaire à peine ou pas différenciée, cette onchocerque s'apparente à la lignée qui comprend l'onchocerque humaine et les onchocerques nodulaires des Bovinés (Bain et Beveridge, 1979). Mais la + présente un caractère unique dans cette lignée qui permet de la distinguer aisément : la couche externe de la cuticule n'a pas de côtes transversales et sa surface est totalement lisse en coupe optique longitudinale. Le $\widehat{\jmath}$, dont la longueur et la taille des spicules sont semblables à celles d'O. volvulus (Leuckart, 1893) et $O$. gibsoni Cleland et Johnston, 1910, est 2 fois plus étroit (80-95 $\mu \mathrm{m}$ au lieu de $150-230 \mu \mathrm{m}$ et $145-200 \mu \mathrm{m})$.

Cette onchocerque constitue donc une espèce nouvelle que nous dédions à H. Schulz-Key en reconnaissance de son aide pour l'extraction des spécimens des nodules; nous la nommons $O$. schulzkeyi $\mathrm{n}$. sp.

\section{2 - Onchocerca hamoni n. sp.}

Hôte: Kobus (Adenota) kob (Erscleben), Reduncinae.

MATÉRIEL : 1 q qui a quelques microfilaires utérines et forme l'holotype et 1 q paratype.

Les deux femelles sont extraites de deux nodules sous-cutanés (dont l'un contenait $1 \hat{\delta}$, perdu par la suite), situées dans la région sacrale d'un des animaux autopsiés.

Description (fig. $2 \mathrm{E}$ et 4 ).

Corps de la femelle mince et rectiligne sur les 1500 premiers microns puis large et enroulé en boucles jusqu'à la queue. Cuticule : couche externe mince sans côtes transversales ni rides longitudinales, parfois un peu ondulée transversalement; couche moyenne épaisse, à stries transversales, couche interne mince. Hypoderme, 
et principalement les cordes latérales, hypertrophié et musculature réduite. Esophage non divisé, inférieur à $1 \mathrm{~mm}$ de long. Vagin à musculature très réduite. Microfilaire mince, à tête ronde et petit crochet.
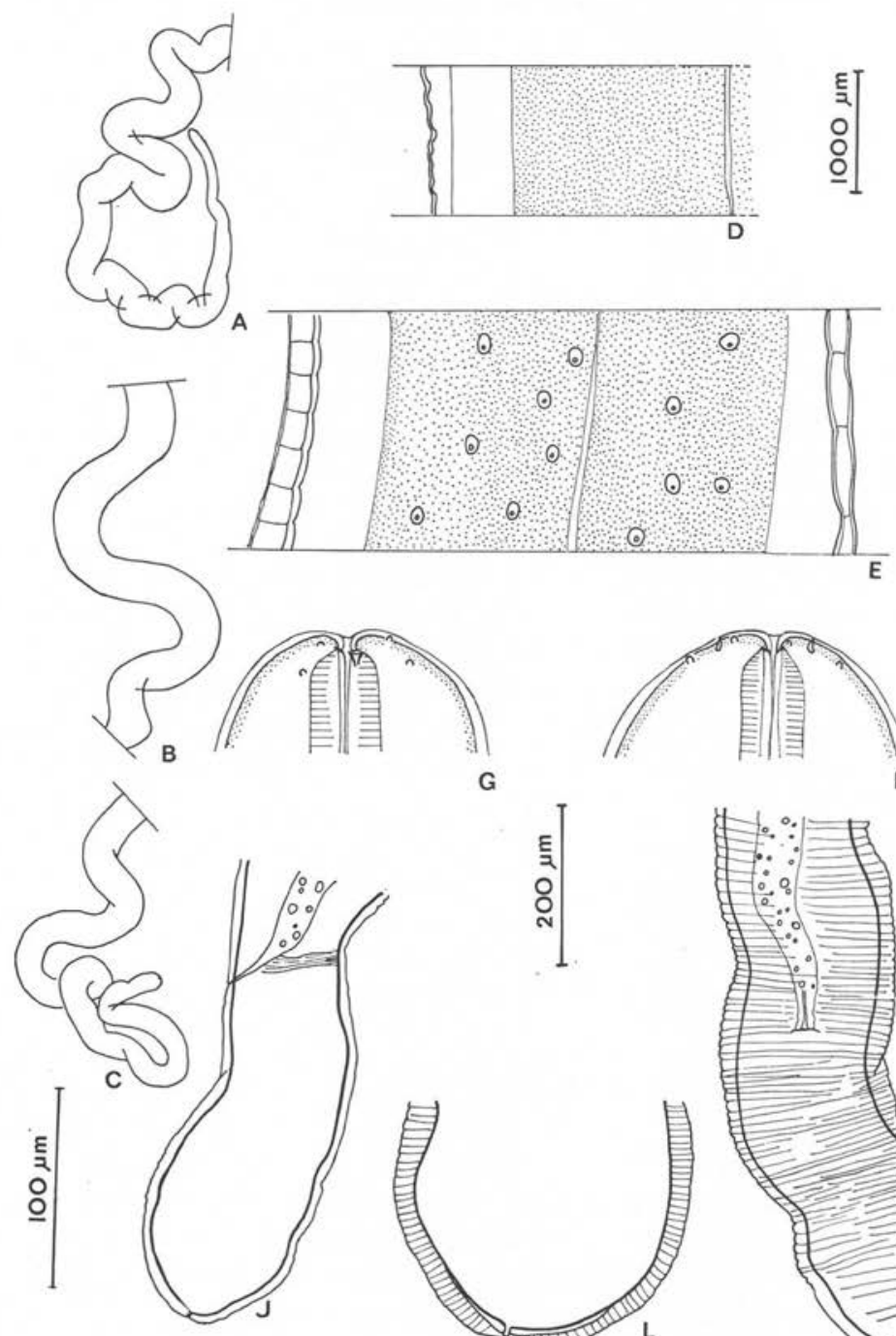

G
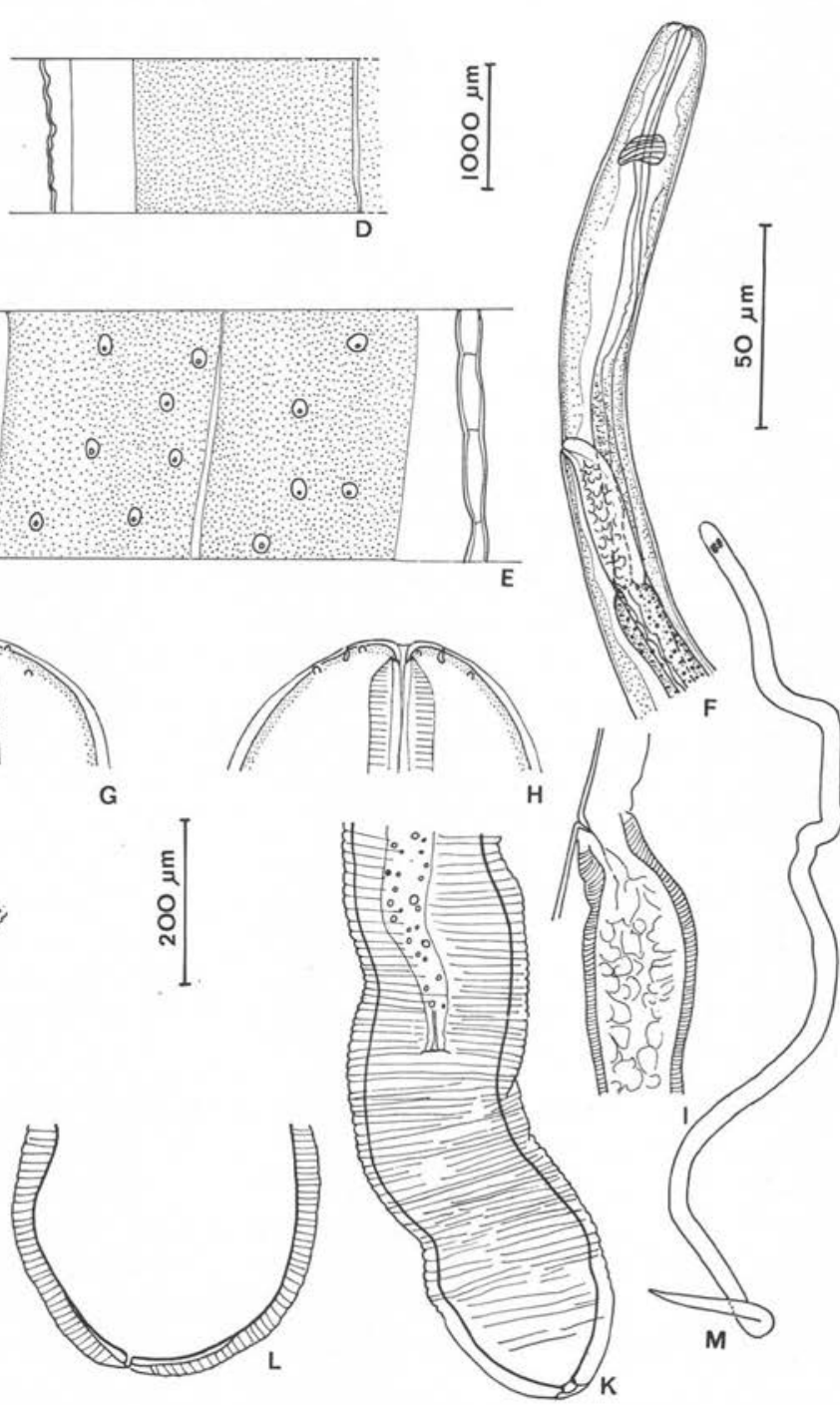

Fig. 4. - Onchocerca hamoni $\mathrm{n}$. sp. femelle; A, B, C: silhouettes des régions antérieures moyenne et postérieure ; D : aspect de la cuticule au niveau de la jonction œsophage-intestin, vue latérale; $\mathrm{E}$ : idem, à mi-corps; $\mathrm{F}$ : région antérieure, vue latérale ; $\mathrm{G}$ et $\mathrm{H}$ : tête, vue latérale et médiane; I. vulve et début de l'ovéjecteur, vue latérale; J. K : queue, vue latérale et ventrale ; L : extrémité caudale, vue latérale ; $\mathrm{M}$ : microfilaire utérine (A, B, C éch. $1000 \mu \mathrm{m}$, J, F : éch. $200 \mu \mathrm{m} ; \mathrm{D}, \mathrm{E}, \mathrm{I}, \mathrm{L}$ éch. Ioo $\mu \mathrm{m} ; \mathrm{M}, \mathrm{G}, \mathrm{H}$ éch. $50 \mu \mathrm{m})$. 
Dimensions 우 holotype : corps long de $350 \mathrm{~mm}$, large de $125 \mu \mathrm{m}$ à la jonction œsophage-intestin, de $270 \mu \mathrm{m}$ à mi-corps et de $200 \mu \mathrm{m}$ au niveau de l'anus; cuticule épaisse de 12 à $22 \mu \mathrm{m}$; stries espacées de 19 à $38 \mu \mathrm{m}$; anneau nerveux et vulve à 185 et $470 \mu \mathrm{m}$ de l'apex ; œsophage long de $855 \mu \mathrm{m}$; queue longue de $460 \mu \mathrm{m}$; microfilaire longue de $250 \mu \mathrm{m}$ et large de $4,8 \mu \mathrm{m}$.

+ paratype : largeur de $90 \mu \mathrm{m}$ à la fin de l'œsophage et de $250 \mu \mathrm{m}$ à mi-corps ; anneau nerveux et vulve à 160 et $550 \mu \mathrm{m}$ de l'apex; cesophage long de $710 \mu \mathrm{m}$; queue longue de $400 \mu \mathrm{m}$.

\section{Discussion.}

Cette onchocerque appartient à la même lignée que l'espèce précédente. Très proche d'O. schulzkeyi n. sp. par la cuticule de la 우 le plus souvent lisse, elle s'en distingue par les dimensions beaucoup plus petites, l'enroulement du corps qui débute plus antérieurement, la couche externe de la cuticule, plus mince et sans rides longitudinales, et la microfilaire plus épaisse $(4,8 \mu \mathrm{m}$ au lieu de $3,5-4 \mu \mathrm{m})$.

O. ochengi Bwangamoi, 1969, est également proche de nos spécimens par la cuticule de la 우 à très faibles ondulations externes transversales, et les dimensions du corps mais la microfilaire est plus grande et plus épaisse $(290-320 \mu \mathrm{m}$ sur 6-7 $\mu \mathrm{m}$ in Bain et coll. 1976, au lieu de $250 \mu \mathrm{m}$ sur $4,8 \mu \mathrm{m})$. Cette onchocerque est donc clairement distincte des autres espèces nodulaires des Bovidés et de l'homme, et bien que le $\sigma^{\star}$ ne soit pas décrit, il nous paraît justifier d'en faire une espèce nouvelle que nous nommons $O$. hamoni n. sp en amical hommage à J. Hamon.

\section{Conclusion}

La récolte de 2 nouvelles onchocerques montre une fois de plus la grande diversité du genre Onchocerca.

Ces deux espèces, très distinctes de la petite lignée des onchocerques de Céphalophinae du Gabon (Bain et coll. 1978), se rattachent à la lignée d'O. volvulus et des onchocerques nodulaires des Bovinés (Bovins domestiques et Syncerus caffer). Il faut probablement rattacher aussi à cette $2^{e}$ lignée 1) les fragments de l'onchocerque récoltée par Le Roux (1947) chez la Céphalope de Grimm, Sylvicapra grimmia (L.), en Zambie, et revue par Bain et Muller (1978), 2) l'onchocerque de l'Élan en Afrique (espèce non précisée) citée par Strong (1935), parce qu'elles sont nodulaires.

Les deux lignées évolutives d'onchocerques actuellement connues chez les Bovidae africains paraissent donc liées, non pas à la sous-famille des hôtes, mais à la région géographique : une lignée assez primitive et non nodulaire dans le bloc forestier du Congo, une lignée nodulaire, aux caractères plus évolués, dans les savanes qui entourent ce bloc (cf. cartes des distributions des hôtes in Dorst et Dandelot 1972). 
Par ailleurs, le fait que les 2 nouvelles onchocerques décrites ici appartiennent à la lignée d'O. volvulus peut avoir des conséquences épidémiologiques.

Il a été récemment montré qu'une onchocerque animale de cette lignée, $O$. ochengi, pouvait être transmise par des simulies du complexe damnosum et qu'il n'était pas possible actuellement de distinguer son stade infestant de celui d'O. volvulus (cf. Denké et Bain, 1978 ; Omar et coll., 1979). Il en résulte des difficultés pour l'évaluation du Potentiel Annuel de Transmission qui constitue le meilleur élément pour juger de l'intensité de l'endémie. Il est possible que ces 2 nouvelles onchocerques compliquent encore le problème.

\section{BIBLIOGRAPHIE}

Bain O., Beveridge I. : Redescription d'Onchocerca gibsoni C. et J. I9Io. Ann. Parasitol., Hum. Comp., 1979, 54, 69-80.

Bain O., Chabaud A. G., Landau I. : Trois nouvelles Onchocerques chez des Céphalophes du Gabon. Ann. Parasitol., Hum. Comp., 1978, 53, 403-419.

Bain O., Denké A. M., Amégée G., Chabaud A. G. : Les onchocerques des Bovins du Togo ; les microfilaires et leurs distributions. Ann. Univ. Bénin, Togo, 1977, 3, 117-123.

BAIN O., Muller R. : Examen de quelques spécimens d'onchocerques animales récoltés par le Dr Le Roux en Afrique Orientale. Ann. Parasitol., Hum. Comp., I978, 53, 3 I I-133.

Bwangamor O. : Onchocerca ochengi new species an intradermal parasite of cattle in East Africa. Bull Epizoot. Dis. Afr., 1969, 17, 321-335.

DenkÉ A. M., Bain O. : Données sur le cycle d'Onchocerca ochengi chez Simulium damnosum s.1. au Togo. Ann. Parasitol., Hum. Comp., 1978, 53, 757-760.

Dorst J., Dandelot P. : Guide des grands Mammifères d'Afrique. Delachaux et Nestlé, Neuchatel (Suisse), 1972, $286 \mathrm{p}$.

LE Roux D. L. : Demonstration : 4, Portion of a female of an Onchocerca sp from a subcutancous nodule in the region of the brisket in a small African antelope, a duiker (Cephalophus grimmi) at Nteko, Isoka district, N. Rhodesia. Trans. Roy. Soc. Trop. Med. Hyg., 1947, 41, 8.

Omar M. S., Denké A. M., Raybould J. N. : The development of Onchocerca ochengi (Nematoda : Filarioidea) to the infective stage in Simulium damnosum s.l. with a note on the histochemical staining of the parasite. Tropenmed Parasit., 1979, 30, 157-162.

Strong R. P. : Onchocerciasis with special reference to the secundary disturbances. Trans. Ass Am. Phys., 1935, 1, 257-261. 